\title{
$P$-手性膦配体促进的手性药物高效合成
}

\author{
许容华 ${ }^{a}$ 杨 贺*,c 汤文军*,a,b,d \\ $\left({ }^{a}\right.$ 上海科技大学物质科学与技术学院 上海 201210) \\ $\left({ }^{b}\right.$ 中国科学院上海有机化学研究所 生命有机化学国家重点实验室 上海 200032) \\ ( ${ }^{c}$ 南方科技大学 深圳格拉布斯研究院 广东深圳 518055) \\ ( ${ }^{d}$ 国科大杭州高等研究院化学和材料科学学院 杭州 310024)
}

\begin{abstract}
摘要 发展高效实用的不对称催化反应对手性药物的简洁合成具有重要的意义, 而手性配体和催化剂对于发展高效的 不对称催化反应尤为关键. 综述了基于苯并氧杂膦烷结构的 $P$-手性单膦和双膦配体的设计理念以及它们在手性药物合 成中的应用. 这类配体具有 $P$-手性、刚性, 大位阻和富电子等结构特征, 物理化学性质稳定, 并且易于衍生化, 在过渡 金属催化的不对称氢化、偶联、环化和加成反应中表现出优异的催化性能. 这些不对称催化反应的发展促进了一系列 重要药物分子的高效不对称合成.
\end{abstract}

关键词＼cjkstart手性膦配体；苯并磷氧杂环；不对称氢化；不对称偶联；手性药物

\section{Efficient Synthesis of Chiral Drugs Facilated by P-Chiral Phosphorus Ligands}

\author{
$\mathrm{Xu}$, Ronghua ${ }^{a} \quad{\text { Yang, } \mathrm{He}^{*, c} \quad \text { Tang, Wenjun }}^{*, a, b, d}$ \\ ( ${ }^{a}$ School of Physical Science and Technology, ShanghaiTech University, Shanghai 201210) \\ ( ${ }^{b}$ State Key Laboratory of Bio-Organic and Natural Products Chemistry, Center for Excellence in Molecular Synthesis, \\ Shanghai Institute of Organic Chemistry, Chinese Academy of Science, Shanghai 200032) \\ ( ${ }^{c}$ Shenzhen Grubbs Institute, Southern University of Science and Technology, Shenzhen, Guangdong 518055) \\ $\left({ }^{d}\right.$ School of Chemistry and Material Sciences, Hangzhou Institute for Advanced Study, \\ University of Chinese Academy of Sciences, Hangzhou 310024)
}

\begin{abstract}
Development of efficient and practical asymmetric catalytic reactions plays a pivotal role for the concise syntheses of chiral drugs. Chiral ligands and catalysts are crucial for the selectivity and reactivity of the catalytic reactions. In this account, the design and development of a series of $P$-chiral mono- and bis-phosphorus ligands were summarized based on a benzooxaphosphane backbone and their applications in the synthesis of chiral drugs. Beside their $P$-chirality, these ligands are structurally rigid, sterically bulky, and electron-rich, providing good physical properties and tunabilities. Facilitated by these chiral ligands, a series of efficient and practical reactions including asymmetric hydrogenation, asymmetric cross-coupling, asymmetric cyclization, and asymmetric nucleophilic additions have been developed. The excellent conversions, yields, regioselectivities, enantioselectivities, and broad substrate scope have enabled concise and efficient syntheses of a series of chiral drugs.

Keywords chiral phosphorus ligands; transition metal catalysis; asymmetric synthesis; enantioselectivity; chiral drugs
\end{abstract}

手性药物 ${ }^{[1]}$ 是指分子结构中含有手性中心或者不对 称中心的药物. 它包括单一的立体异构体、两个或两个 以上立体异构体的混合物. 由于受体、酶或离子通道具 有高度的立体结构特异性, 所以手性药物的不同立体异
构体与靶点的相互作用有所不同，从而产生不同的药理 学活性、代谢过程及生物毒性. 包含 “反应停” 在内的 一系列医学事件深刻表明获取光学纯药物以及对其立 体异构体的研究在疾病研究和药物发展中尤为重要. 因

\footnotetext{
* Corresponding authors. E-mail: yanghe@sioc.ac.cn; tangwenjun@sioc.ac.cn Received March 6, 2020; revised April 6, 2020; published online April 17, 2020.

Project supported by the National Natural Science Foundation of China (Nos. 21725205, 21432007, 21572246), the Strategic Priority Research Program of the Chinese Academy of Sciences (XDB20000000) and the K. C. Wong Education Foundation.

国家自然科学基金(No. 21725205, 21432007, 21572246)、中国科学院战略性先导科技专项(XDB20000000)和王宽诚教育基金会资助项目.
} 
此, 1992 年美国食品药品监督管理局(FDA)规定, 新的 手性药物上市之前必须分别对左旋体和右旋体进行药 效和毒性试验, 否则不允许上市. 2006 年 1 月, 我国国 家食品药品监督管理总局(SFDA)也出台了相应的政策 法规.

在我们常用的小分子药物中, 叔(季)碳手性中心、 甾体、 $\beta$-芳基胺、三级醇、三级胺的结构单元是广泛存 在的(图 1), 例如 indacrinone 是具有手性叔碳中心结构 单元的降血压药物; 用于性激素调节的甾体类药物 ethinylestradiol 含有五个手性中心; bortezomib 是典型的 具有手性 $\beta$-芳基胺结构单元的药物, 其可以用于治疗多 发性骨髓瘤和套细胞淋巴瘤; clemastine 是目前世界上 公认的最好的抗组胺药之一, 其含有手性二芳基三级醇 的结构; 具有连续两个手性中心和三级胺结构的药物分 子 tofacitinib 是治疗类风湿关节炎、溃疡性结肠炎、银 屑病等多种炎症的实用药物; 具有轴手性的右旋联苯双 酯可用于治疗病毒性肝炎和药物性肝损伤引起转氨酶 升高症状.

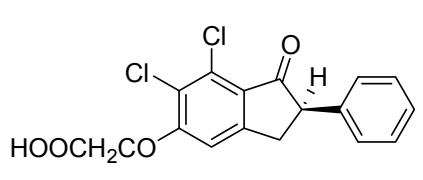

Indacrinone<smiles>CCCC(NC(=O)C(Br)NC(=O)c1cnccn1)C(=O)O</smiles>

Bortezomib<smiles>C[C@@H]1CCN(C(=O)CC#N)C[C@H]1N(C)c1ncnc2[nH]ccc12</smiles>

Tofacitinib

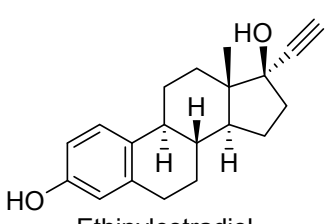

Ethinylestradiol

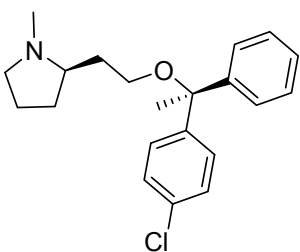

Clemastine

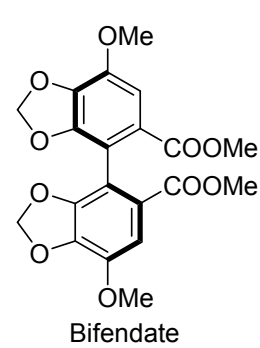

图 1 一些含有手性结构单元的畅销和常用药物

Figure 1 Some popular and commonly used drugs with important chiral units

发展高对映选择性、高产率和原子经济性的催化反 应对手性药物的高效合成至关重要. 目前构建分子的手 性中心一般有如下的一些方法: 外消旋体的拆分, 使用 手性试剂(当量级使用), 手性助剂参与的不对称合成、 不对称催化等 ${ }^{[2]}$. 其中不对称催化, 尤其是过渡金属催 化的不对称反应具有底物普适性广、原子经济性强、环
境耐受性佳、反应容易放大等特点，决定了过渡金属催 化在手性药物合成中的应用潜力. 手性配体的特性, 包 括电子效应、立体效应和空间效应等直接影响了催化剂 的活性和立体选择性. 设计发展具有新型骨架的配体和 催化剂是解决过渡金属催化的不对称反应中高效性和 选择性的重要途径. 近年来, 手性膦配体在不对称催化 领域得到了长足的发展，并广泛应用于手性药物分子的 合成中. $P$-手性膦配体具有显著结构特征, 表现出独特 的催化特性和应用前景. 图 2 列举了一些代表性的 $P$-手 性单膦和双膦配体 ${ }^{[3]}$. 本文将综述我们研究小组在基于 苯并氧杂膦烷刚性骨架的 $P$-手性膦配体的设计和发展、 $P$-手性膦配体促进的高效不对称反应方法学和最终应 用于手性药物的高效合成方面的研究思路和实践 ${ }^{[4]}$.

monophosphorus ligand

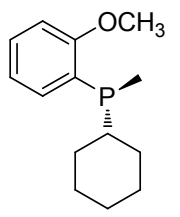

CAMP

bisphosphorus ligand<smiles>CCC(C)PCP(C)CP(C)CC</smiles>

MiniPhos<smiles>COc1cccc(OC)c1-c1cccc2c1P(CBr)CO2</smiles>

BI-DIME

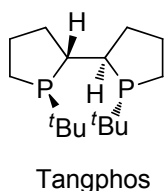<smiles>CC(C)(C)P1COc2cccc(-c3c4ccccc4cc4ccccc34)c21</smiles>

AntPhos
图 2 一些典型的 $P$-手性的单膦和双膦配体

Figure 2 Selected examples of typical $P$-chiral mono- and bisphosphine ligands

\section{1 新型手性膦配体的设计与发展}

手性配体的结构对过渡金属催化的不对称反应的 活性和立体/对映选择性有至关重要的影响. 苯并氧杂 膦烷是具有刚性的结构单元，基于此类骨架的配体有发 展成为优势配体的潜力(图 3). 当苯并氧杂膦烷中的磷 原子上含有大位阻取代基(叔丁基)时，所发展的配体往 往具有稳定的 $P$-手性. 这些光学活性纯的 $P$-手性单膦 和双膦配体在过渡金属催化的不对称反应中往往表现 出独特的催化特性 ${ }^{[5]}$. 具体来讲, 单膦配体 I 具有以下 特点(图 3): (1)配体中的苯并氧磷杂五元环的刚性结构 使其具有确定的构象，这保证了在催化反应中催化剂与 底物作用时具有确定的优势面和劣势面，从而显著提高 催化反应的活性和立体/对映选择性; (2)磷原子上的供 电子取代基使这类配体更加富电子，同时具有 $P$-手性 的配体的手性因素更加靠近过渡金属中心，因而含有这 种配体的催化剂有更高的催化效率和更好的手性诱导; (3)通过改变配体中 $\mathrm{R}^{1} 、 \mathrm{R}^{2}$ 以及下面芳环的结构可以对 配体的电性及位阻进行调控，从而优化配体的催化活性 
和选择性; (4)单膦配体 $\mathbf{I}$ 的联芳基结构以及叔丁基取代 基增大了磷原子周围的位阻，使得这类配体具有优良的 化学稳定性, 增大了操作的简便性和实用性.

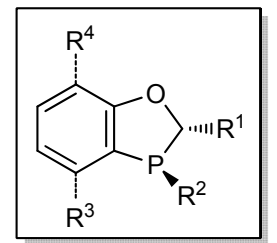

- Five-membered oxaphosphole ring has a defined conformation

- Benzene ring improves physical properties and chemical stability

- Functionalization at different positions allows high tunability

- Bulky substituents leads to configurationally stable P-chirality

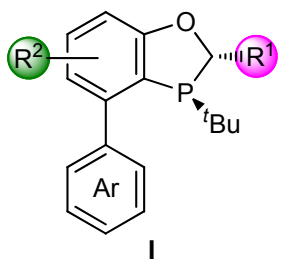

monophosphorus ligand

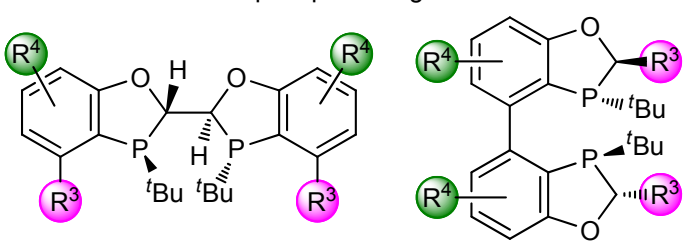

II: BIBOP-type bisphosphorus ligand

图 3 基于苯并氧杂膦烷结构的配体类型

Figure 3 Ligand structures based on chiral benzooxaphosphane skeleton
基于苯并氧磷杂五元环刚性骨架的双膦配体有两 种类型：具有 $C_{2}$ 对称性的 BIBOP 类型配体 II 和具有 $C_{2}$ 对称性的联芳基 BABIBOP 类型配体 III. BIBOP 类型 配体 II 是基于双膦配体 TangPhos 发展而来(图 4). 和 TangPhos 相比，芳环的引入使这一类配体具有更好的空 气稳定性. 由于能够通过拆分手段获得光学纯的配体骨 架, BIBOP 类型配体 II 的合成又更加简便. 由于具有联 芳基结构, BABIBOP 类型配体 III 有着和 BIBOP 类型配 体 II 截然不同的空间构型，因而也表现出和配体 II 互补 的催化活性. 值得指出的是, 手性双膦配体 II 和 III 同 样具有结构和电性可调的特点, 通过改变配体中 $\mathrm{R}^{3}$ 或 $\mathrm{R}^{4}$ 基团可以有效地对配体的催化活性进行优化 ${ }^{[5]}$.
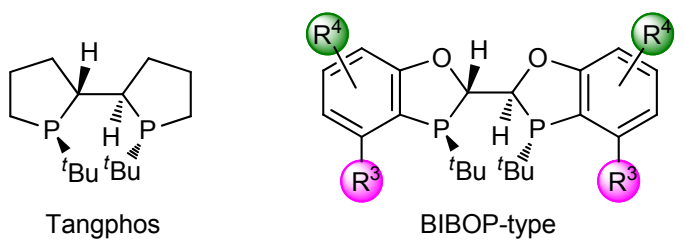

图 4 Tangphos 和 BIBOP 的结构

Figure 4 Structures of Tangphos and BIBOP

苯并氧杂膦烷的合成路线如 Scheme 1 所示. 最初 的路线以甲基二氯膦(1)为起始原料，其与叔丁基格氏 试剂反应, 然后用锂化的 1,3-二甲氧基苯和 $\mathrm{H}_{2} \mathrm{O}_{2}$ 连续处 理, 可以以 $80 \%$ 的收率得到氧化膦 2 . 在 ${ }^{n} \mathrm{BuLi} / \mathrm{I}_{2}$ 的条件 下，在 2 的 $\alpha$ 位上进行碘化，以 $80 \%$ 的收率合成化合物 3. 用 $\mathrm{BBr}_{3}$ 对甲氧基进行脱保护, 然后在碱性条件下闭 环，可以以 $90 \%$ 的收率得到关键结构单元 4. 整个过程
A

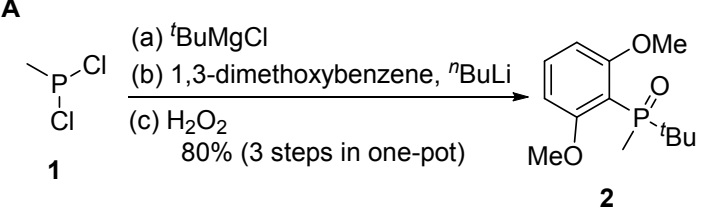

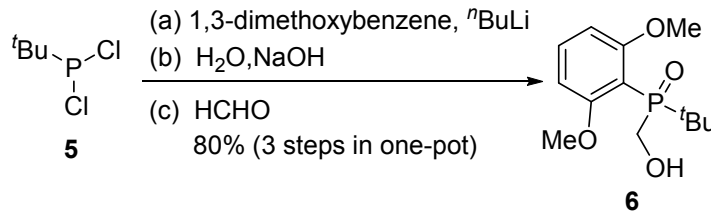

$\frac{{ }^{n} \text { BuLi, TMDEA }, \mathrm{I}_{2},-78^{\circ} \mathrm{C}}{80 \%}$

(a) $\mathrm{HI},-78^{\circ} \mathrm{C}$ (b) $\mathrm{MsCl}, \mathrm{Et}_{3} \mathrm{~N}$

(c) $\mathrm{NaOH}$ $88 \%$ (3 steps)

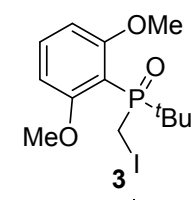

(a) $\mathrm{BBr}_{3} \mid 90 \%$

(b) $\mathrm{K}_{2} \mathrm{CO}_{3}$ (2 steps)

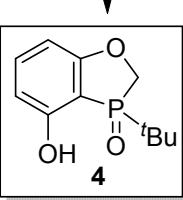

resolution with (+)-menthyl chloroformate

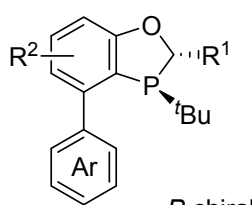

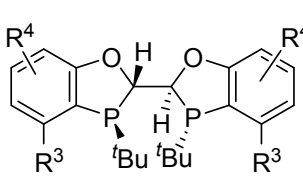

$\mathrm{R}^{3} \quad{ }^{t} \mathrm{Bu}{ }^{t} \mathrm{Bu} \quad \mathrm{R}^{3}$

$\mathrm{R}^{4}$

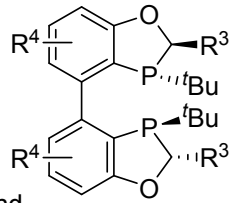

图式 1 苯并手性氧杂膦烷结构单元的合成路线

Scheme 1 Synthetic route of chiral benzooxaphosphane unit 
料, 经过锂化的 1,3-二甲氧基苯、水和甲醛处理, 可以 以 $80 \%$ 的分离产率得到 6 . 用 $\mathrm{HI}$ 进行甲氧基脱保护，然 后形成甲磺酸酯和闭环得到 4. 以手性氯甲酸薄荷酯为 试剂对 4 进行拆分, 可以高效地得到光学纯的 7, 分离 产率为 $42 \%$. 以该手性结构单元为关键合成中间体, 合 成了一系列具有 $P$ 手性的单膦和双膦配体 ${ }^{[5]}$.

图 5 中列出了常用的基于苯并氧杂膦烷结构的 $P$ 手性单膦和双膦配体, 其中单膦配体 $\mathbf{L 1} \sim \mathbf{L 5}$ 在过渡金 属钯催化的不对称 Suzuki-Miyaura 偶联和分子内去芳构 环化反应中显示出优异的催化特性. 我们利用相应的方 法学实现了布洛芬、氟比洛芬、菜普生、雷公藤对醌 $\mathrm{H}$ 和包括棉酚在内的联芳基药物的合成. 手性双膦配体 L6 L8 被应用于 $\mathrm{Rh}$ 催化的不对称氢化和加成反应中， 表现出高立体选择性和反应效率. 因此实现了托法替 尼、艾司西酞普兰和 cipargamin 的高效合成.

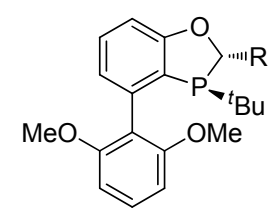

$\mathrm{R}=\mathrm{H} \quad(\mathrm{S})-\mathrm{BIDIME}(\mathbf{L} \mathbf{1})$ $\mathrm{R}={ }^{i} \operatorname{Pr}(\mathrm{L} 2)$

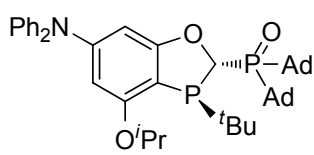

L4

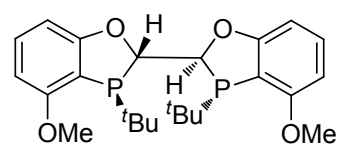

(S,S,S,S)-MeO-BIBOP (L6)

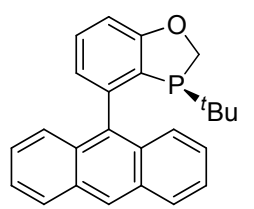

(S)-AntPhos (L3)

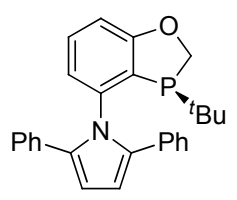

L5

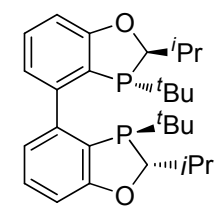

L7

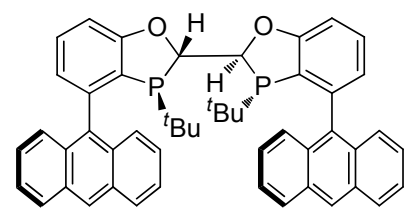

$(S, S, S, S)$-WingPhos (L8)

图 5 常用的 $P$-手性单膦和双膦配体

Figure 5 Commonly used $P$-chiral mono- and bis-phosphorus ligands

\section{2 不对称催化方法学和手性药物合成}

按照不同的反应类型阐述 $P$-手性膦配体所表现出 的独特反应效率和选择性, 以及它们在手性药物合成中 的成功应用.

\section{1 不对称氢化合成西洛多辛中间体和托法替尼}

三取代烯酰胺的不对称氢化是一种制备手性氨基
酸和 $\beta$-芳基胺的理想的方法. 虽然目前此方面的研究有 许多报道，并且获得了高对映选择性，但是提升手性催 化剂的转化数(TON), 即实现在低催化剂用量条件下的 不对称氢化仍然面临巨大的挑战. 为了解决这一难题, 我们设计并合成了含有深口袋的手性双膦配体 WingPhos (L8), 期望可以通过改变金属与配体的配位模式与 过渡态结构来提高催化剂的活性和稳定性(图 6). 配体 L8 具有以下几个优势: (1)与目前常用于不对称氢化的 BINAP 和 DuPhos 等配体相比, WingPhos 具有截然不同 的构象，所形成的金属催化剂也有不同的手性环境，从 而表现出与 BINAP 和 DuPhos 等配体互补的催化性能; (2)由于两个葱基的存在, WingPhos 具有深且构象确定 的口袋，可以实现远程手性诱导；(3) WingPhos 易于制 备且在空气中稳定, 具有工业应用的潜力. 研究发展, $\mathrm{Rh} / \mathbf{L 8}$ 催化剂可以使三取代 $\beta$-芳基烯酰胺在温和条件下 发生不对称氢化, 得到一系列含有不同官能团的手性 $\beta$ 芳基酰胺化合物. 在底物结构中, 除了链状三取代的烯 酰胺外, 氢化反应还可以兼容环状三取代的烯酰胺化合 物. 值得指出的是, 在三取代 $\beta$-芳基烯酰胺的不对称氢 化反应中, $\mathrm{Rh} / \mathbf{L 8}$ 催化剂的转化数达到 10000 , 同时反应 的转化率和对映选择性不受影响. 这些结果表明 WingPhos 具有很大的工业应用前景，可用于许多含有 $\beta$-芳基胺结构单元的药物分子和天然产物的合成中 ${ }^{[6]}$.

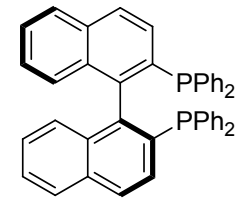

(S)-BINAP

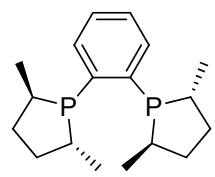

$(R, R)$-Me-DuPhos

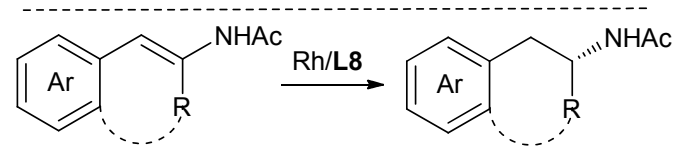

up to $99 \%$ yield 93\% 99\% ee up to 10000 TON
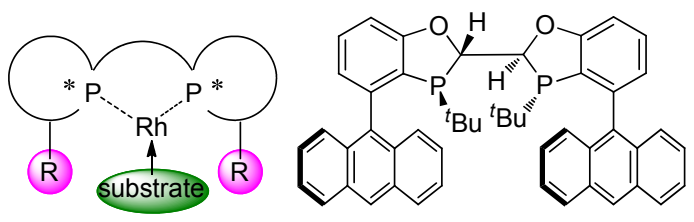

$(S, S, S, S)$-WingPhos (L8)

图 $6 \mathrm{Rh}$ 催化的烯酰胺的不对称氢化

Figure 6 Rh-catalyzed asymmetric hydrogenation of enamides

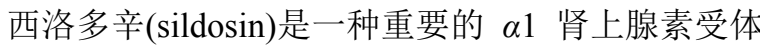
拮抗剂, 主要用于治疗与良性前列腺增生或肥大相关的 泌尿系统疾病. 其分子内含有 $\beta$-芳基手性仲胺的结构可 以通过相应烯胺衍生物的不对称氢化合成. 如 Scheme 2 
所示, 烯酰胺化合物 9 可以通过易于制备的硝基苯乙烯 衍生物还原得到. 在 $\mathrm{Rh} / \mathrm{L8}$ 催化剂的存在下, 9 经过高对 映选择性氢化反应转化为手性酰胺 $\mathbf{1 0}$. 参照文献报道 的步骤可以将此关键中间体 10 转化为西洛多辛.

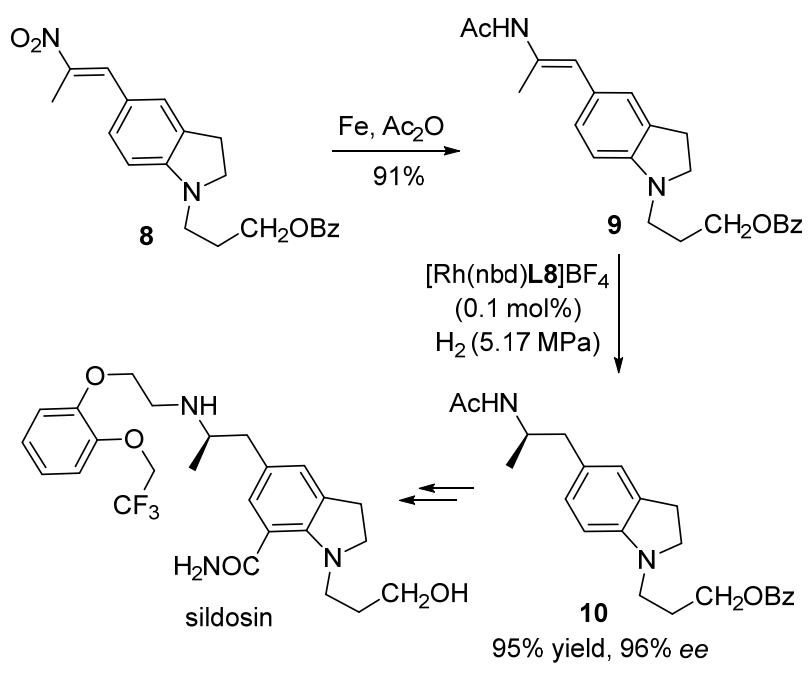

图式 2 Sildosin 的关键中间体的合成

Scheme 2 Synthesis of the key intermediate of sildosin

和三取代烯酰胺的不对称氢化相比, 四取代烯酰胺 的不对称氢化更具有挑战性. 一方面, 取代基的增多导 致烯酰胺底物的位阻增大, 降低了氢化反应的活性, 并 使得催化剂的用量增加. 另一方面, 目前所发展的配体 或催化剂往往适用于某种特定结构的底物的氢化, 官能 团和底物结构的兼容性较差. 为了解决这些难题并发展 普适性强的四取代烯酰胺的不对称氢化方法, 认为配体 的设计与发展至关重要. 在发展了深口袋的 Wing-Phos 的基础上, 设想通过提高配体整体的富电性来增强配体 的催化活性. 同时通过引入手性的深口袋来增强催化剂 对氢化反应的手性诱导, 继而提高反应的对映选择性. 基于富电子的 BIBOP 型骨架设计合成了一系列新型的 含有手性深口袋的双膦配体. 在环状四取代烯酰胺的不 对称氢化中, 发现使用 ArcPhos 可以得到优异的产率和 对映选择性. 经过克级规模试验发现, 催化剂 Rh/L9 最 高的转化数可以达到 10000 , 具有很好的实用前景 ${ }^{[7]}$. 通过 ${ }^{31} \mathrm{P} N M R$ 、配体与金属的金属络合物的 XRD 单晶 衍射以及计算化学的辅助手段, 提出了可能的催化循环 (图 7). 该不对称氢化的反应路径是经过 “dihydride” 的 过程(C), 而对映选择性的决定步骤在于酰胺的双键与 $\mathrm{Rh}$ 金属中心的配位过程(D vs E). 反应经能量最有利的 过渡态 $\mathbf{E}$ 发生 $\mathrm{Rh}-\mathrm{H}$ 对底物双键的插入和后续还原消 除给出相应的手性产物. 利用这个不对称氢化作为关键 步骤, 我们实现了药物分子托法替尼的合成.

托法替尼是用于治疗类风湿性关节炎、银屑病关节
炎和溃疡性关节炎的重要药物 ${ }^{[7]}$. 其是由辉瑞公司针对 免疫抑制作用所开发的一种小分子 Janus 激酶(JAK)抑 制剂. Janus 激酶是四个与受体相关的酪氨酸激酶 (JAK1-3, TYK2)的家族, 主要参与包括造血和免疫反应 等一系列生理过程. 托法替尼最近被美国食品药品监督 管理局批准用于治疗类风湿性关节炎. 由于其显示出一 些不利的副作用，欧洲药品管理局目前尚未批准. 托法 替尼主要是通过对其外消旋体的手性拆分来获得的 ${ }^{[8]}$. 目前所报道的两例关于托法替尼的不对称合成路线效 率很低 ${ }^{[9]}$, 不适于该化合物的大量制备. 为了发展一条 高效的不对称合成托法替尼的路线，可以通过四取代烯 酰胺的不对称氢化策略来实现托法替尼的高效不对称 合成(Scheme 3). 具体地, 托法替尼可以通过中间体 11 和 12 经过芳环的亲核取代得到. 中间体 12 则可以通过 酰胺 13 经酰基保护基脱除和两次烷基化得到. 容易制 备的环状烯酰胺 14 可通过不对称氢化转变为手性酰胺 13.

基于对托法替尼的反合成分析，我们参照已报道的 方法很容易地合成了烯酰胺底物 14 (Scheme 4). 使用 $\mathrm{Rh} / \mathbf{L 9}$ 催化剂对 $\mathbf{1 4}$ 进行不对称氢化, 手性酰胺 $\mathbf{1 3}$ 可以 以 $99 \%$ 的收率和 $96 \%$ ee 顺利得到. 这一化合物的绝对 构型通过 $\mathrm{X}$ 射线晶体学分析确定. 在 $\mathrm{Li} / \mathrm{NH}_{3}$ 条件下, 13 的三氟甲磺酰基保护基顺利脱除，接着经苄基引入，脱 乙酰基保护基和甲基化步骤顺利转化为 12.11 和 12 在 碳酸钾的条件下进行偶联, 并经后续苄基脱除以及与氰 基乙酸的缩合反应得到 $(R)$-托法替尼. 值得指出的是, 在所有转化中, 产物的光学纯度没有任何损失. 我们最 终实现了 $(R)$-托法替尼的克级规模合成.

\section{2 不对称加成反应合成 Cipargamin 和艾司西酞普兰}

手性 $\alpha$-三级胺结构广泛存在于许多药物分子中. 不 对称 Strecker 和 Mannich 型反应是合成特定类型的手性 $\alpha$-三级胺的重要方法, 但是这两类反应不能用来合成 $\alpha, \alpha$-二芳基三级胺这类重要的手性片段. 在早期, 过渡 金属催化的烷基锌试剂对酮亚胺的不对称加成成为获 得手性 $\alpha$-芳基-三级胺的方法之一，也为手性 $\alpha, \alpha$-二芳 基三级胺的合成提供了思路. 相比于金属试剂具有制备 繁琐、环境敏感、官能团兼容性不好等缺点，有机硼试 剂往往具有简单易得和稳定性高的明显优势. 因此, 芳 基嗍试剂对亚胺的不对称亲核加成是一种合成手性 $\alpha, \alpha$ 二芳基三级胺的便捷方法. 但目前报道的方法都要先将 亚胺进行保护，然后发生加成反应，最后再进行脱保护. 通常这些脱保护条件都很剧烈, 官能团容忍性较差. 因 此, 发展一个芳基硼试剂对无保护亚胺的不对称亲核加 成反应具有重要的意义. 我们基于 BIBOP 配体骨架进 一步对配体结构进行了优化, 成功发展了配体 L10. 最 


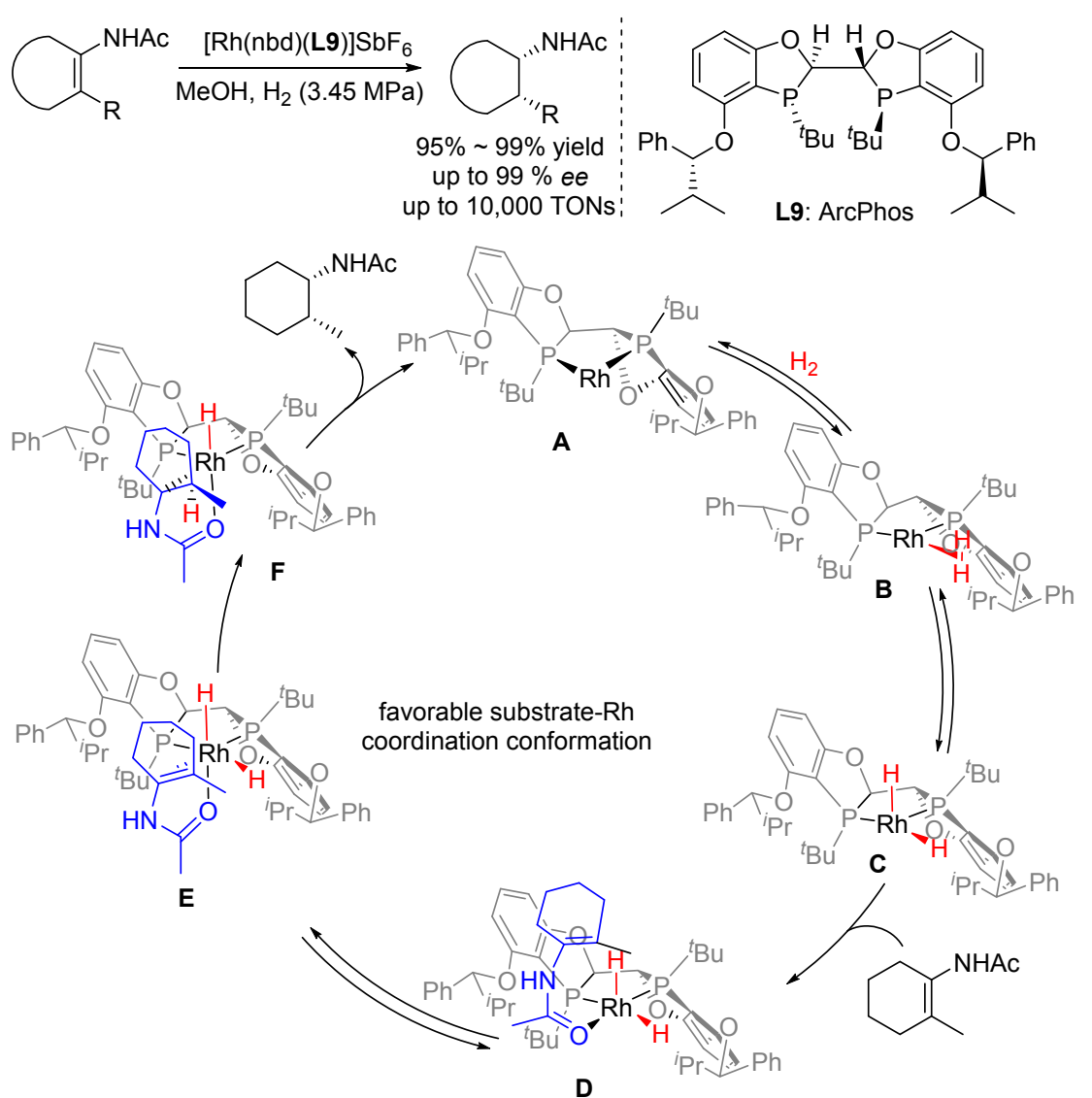

图 7 四取代烯酰胺的不对称氢化: 催化循环

Figure 7 Proposed catalytic cycle for asymmetric hydrogenation of tetra-substituted enamides<smiles>C[C@@H]1CCN(C(=O)CC#N)C[C@H]1C(=O)N(C)c1ncnc2[nH]ccc12</smiles>

$(R)$-tofacitinib<smiles>CCNC1=C(C)CN([Tl])CC1</smiles>

Readily available

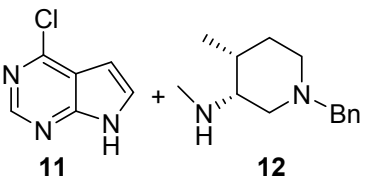

$\Downarrow$

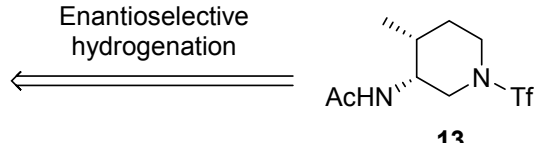

13

图式 3 托法替尼的逆合成分析

Scheme 3 Retrosynthetic analysis of tofatinib

终实现了 $\mathrm{Rh} / \mathbf{L 1 0}$ 催化的芳基硼酸酐对靛红衍生的 $\mathrm{N}-$ $\mathrm{H}$ 亚胺的不对称加成反应(Scheme 5). 该方法学的底物 普适性好，可以兼容含有不同取代基和位阻的底物. 利 用该方法学可以以优秀的收率和对映选择性合成一系 列手性 3-氨基-3-芳基吲哚酮结构 ${ }^{[10]}$.

Cipargamin 是诺华制药 Thierry Diagana 等 ${ }^{[10]}$ 开发的 用于治疗㾏疾的潜在药物. 其具有很好的药代动力学性 质, 在啮齿动物模型中有很好的抗疮疗效 ${ }^{[11]}$. 目前该化
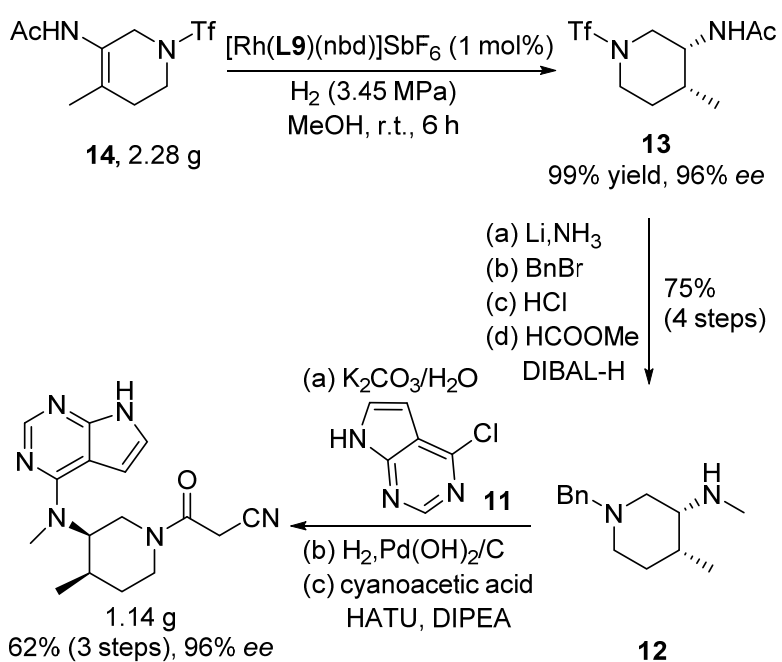

图式 4 托法替尼的不对称合成

Scheme 4 Asymmetric synthesis of tofatinib

合物已经进入临床二期的研究. 经过对这个分子的逆合 成分析, 我们认为其哌啶环可以通过手性三级胺的分子 内还原胺化构建(Scheme 6). 而化合物 16 结构中的手性 三级胺基团可以方便地利用芳基硼试剂 17 对取代的靛 红亚胺 18 的对映选择性加成合成得到. 


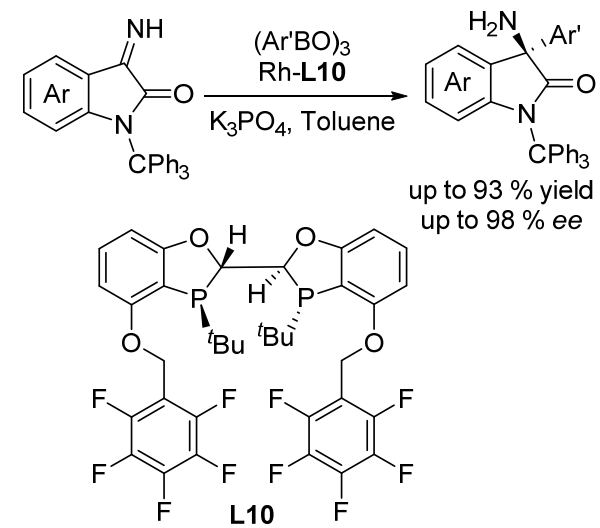

图式 $5 \mathrm{Rh}$ 催化的芳基硼酸酐对无保护亚胺的不对称亲核加 成

Scheme 5 Asymmetric nucleophilic addition of arylboronic anhydride to unprotected imine<smiles>CC1=C(C)N[C@@]2(C1)C(=O)Nc1ccc(F)cc1[C@@H](C)Cc1c2[nH]c2cc(Cl)c(Cl)cc12</smiles>

15

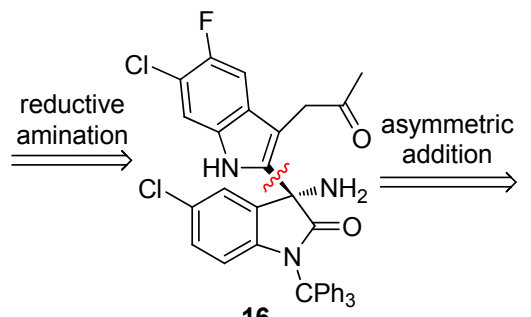

16<smiles>CC(=O)Cc1c(Br)[nH]c2cc(Cl)c(F)cc12</smiles>

17<smiles>[13CH3]N1C(=O)C(=N)c2cc(Cl)ccc21</smiles>

图式 6 Cipargamin 的逆合成分析

Scheme 6 Retrosynthetic analysis of cipargamin

在实际的合成过程中, 我们利用已知化合物吲哚硼 酯 19 和亚胺 18 作为起始原料, 使用 $\mathrm{Rh} / \mathbf{L 1 0}$ 催化剂成 功实现了 19 对 18 的不对称加成反应, 以 $65 \%$ 的产率和 96\%的 $e e$ 值顺利得到手性胺 20 (Scheme 7). 接着经过脱 除缩酮保护基和立体选择性分子内还原胺化, 20 顺利转 化成螺环结构 21, 最后脱除三苯甲基保护基成功地合 成 cipargamin.

除了对酮亚胺的不对称加成合成手性 $\alpha$-芳基三级 胺外, 芳基硼酸或硼酸酐对酮的不对称加成反应则是构 建手性叔碳中心的重要方法. 基于手性双膦配体 WingPhos, 我们也成功实现了芳基硼酸酐对芳基酮的 不对称加成反应，同时利用该方法学实现了手性药物分 子艾司西酞普兰(Escitalopram) 的首次对映选择性合 成 ${ }^{[12]}$.

艾司西酞普兰是一种具有高选择性的 5-羟色胺再 摄取抑制剂. 它在治疗中度至重度广泛性焦虑症(GAD) 或社交焦虑症(SAD), 恐慌症(伴或不伴恐惧症)以及强

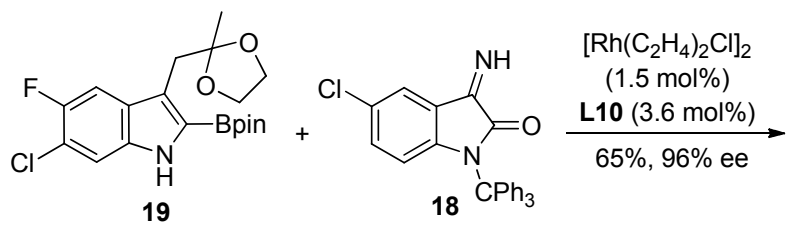<smiles>CC1(Cc2c[nH]c3cc(Cl)c(F)cc23)C[C@@]2(N)C(=O)Nc3ccc(Cl)cc3[C@@H]12</smiles>

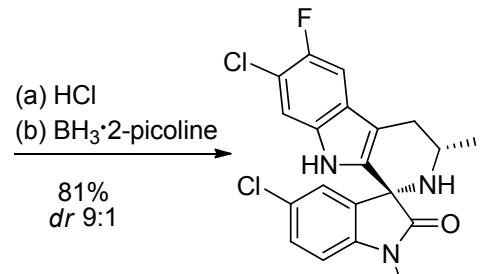

$20 \mathrm{CPh}_{3}$

$21 \mathrm{CPh}_{3}$
图式 7 Cipargamin 的不对称合成

Scheme 7 Asymmetric synthesis of cipargamin

迫症(OCD)方面有很好的效果且普遍耐受. 工业生产艾 司西酞普兰主要依靠外消旋混合物的化学或酶促拆分 实现 ${ }^{[13]}$ ，其总收率和效率都较低. 目前艾司西唒普兰的 高效不对称合成仍然是一个重大挑战. 从合成角度分 析, 我们认为艾司西酞普兰分子内的四氢呋喃环可以通 过叔醇 22 经双氰基化一内酯化-内酯还原构建(Scheme 8). 手性三级醇 22 则可以利用芳基硼试剂对芳基酮 23 的不对称加成得到. 于是研究了 4-氟苯基硼酸酐对芳基 酮 23 的不对称加成反应(Scheme 9). 令人欣慰的是，使 用 $\mathrm{Rh} / \mathrm{L8}$ 催化剂, 我们成功地得到光学纯的加成产物 22 ( $>99 \% e e$ ). 经二甲胺参与的取代反应和钯催化的双氰 基化一内酯化过程，22 以 $73 \%$ 的总收率转化为内酯 25 . 随后 25 经过 DIBAL-H, $\mathrm{MsCl}$ /三乙基胺和 $\mathrm{NaBH}_{4}$ 还原等 处理得到艾司西酞普兰.

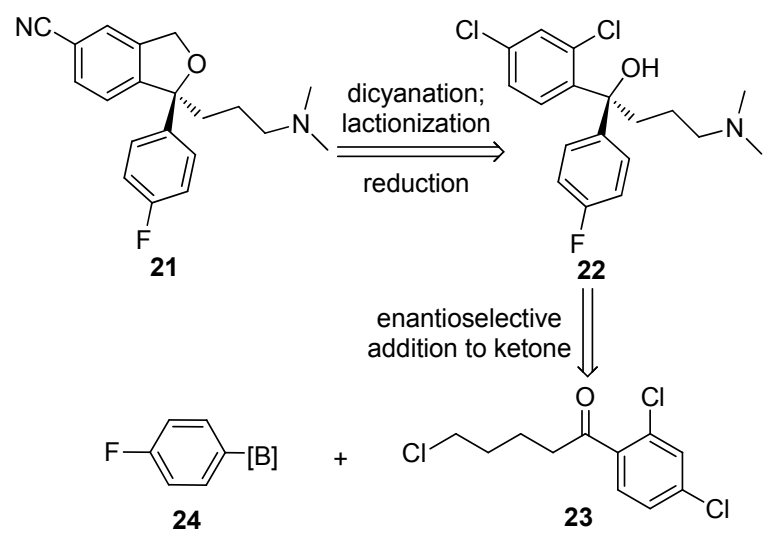

图式 8 艾司西酞普兰的逆合成分析

Scheme 8 Retrosynthetic analysis of escitalopram 

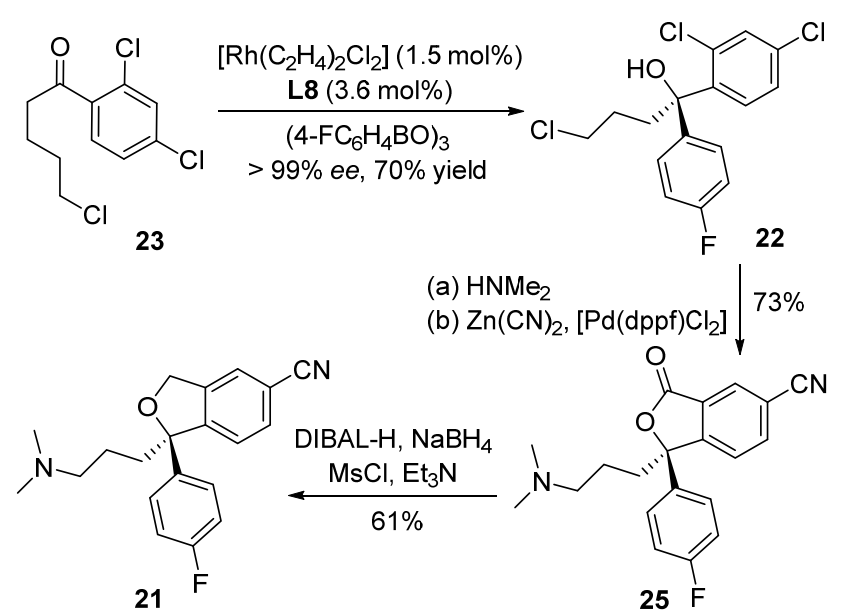

图式 9 艾司西酞普兰的不对称合成

Scheme 9 Asymmetric synthesis of escitalopram

\section{3 不对称去芳构环化合成雷公藤对醌 $\mathrm{H}$}

含手性季碳的并环骨架广泛存在于众多具有生物 活性的萜类和甾体类天然产物和药物分子中. 发展针对 这类含手性季碳多环骨架的合成方法具有非常重要的 意义. 不对称环化是一类高效的构建环系结构骨架的方 法，其中，通过过渡金属催化的不对称去芳构环化是一 种具有吸引力的构建含季碳的多环结构的途径.

我们课题组 ${ }^{[14]}$ 最近发展了一个不对称去芳构环化 反应试图实现一系列萜类、甾体、生物碱和聚酮等具有 重要生物活性分子的高效合成. 我们提出并设计了一个 不对称去芳构环化方法. 如图 8 所示, 结构 26 可以经过 钯催化的分子内两个芳环之间的不对称去芳构偶联而 转化为含有手性季碳中心的并环骨架. 实现这一环化反 应的关键在于如何解决该反应的活性、区域选择性和对 映选择性的问题. 依照我们提出的催化循环, 该反应首 先经过钯对芳基溴 26 的氧化加成可能得到手性膦配体 配位的芳基钯中间体 Ia 或 Ib. 接下来的芳基取代位点 决定了反应的化学选择性. 由钯中间体 Ia 出发的芳基 取代发生在酚羟基对位(即 a 位), 形成去芳构化钯中间 体 IIa, 再经过还原消除得到所需要的含有手性季碳的 去芳构环化产物 27; 而由钯中间体 Ib 出发的芳基取代 发生在酚羟基的邻位(即 b 位)得到 IIb, 最终得到非手性 产物 28. 从可能的反应产物分析, 尽管平面性分子 $\mathbf{2 8}$ 在热力学方面较含有手性季碳三环结构 27 更稳定, 然 而 27 的生成从反应动力学角度来看可能更有利. 当 $\mathbf{L}^{*}$ 是一个大位阻单齿膦配体时, Ib 构象由于存在可能的配 体和酚羟基负离子间作用而变得不稳定, 平衡将向 Ia 构象的生成倾斜, 促使产物 $\mathbf{2 7}$ 的形成. 同时, 膦配体的 手性因素可以对去芳构环化反应进行高效率地诱导, 实 现高对映选择性的转化. 因此, 我们设想利用大位阻单
齿 $P$-手性膦配体来调控该反应的活性和选择性问题. 通过研究配体结构和反应活性/选择性的关系，我们发 现大位阻单膦配体如 AntPhos 可以给出理想的产率和对 映选择性. 该不对称环化反应表现出很好的官能团和底 物结构类型的容忍性.

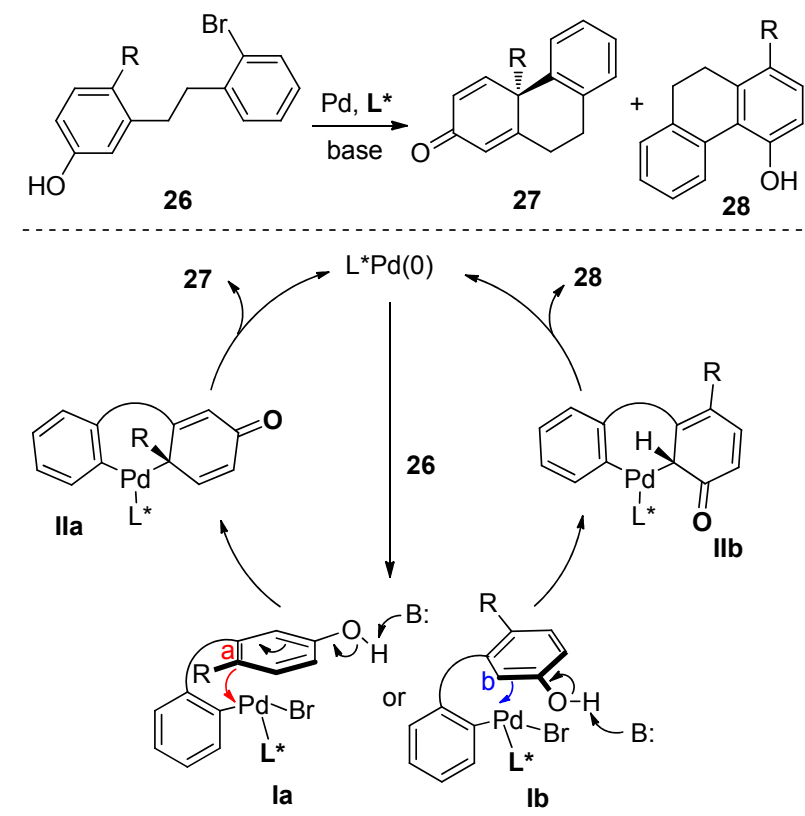

图 8 Pd 催化的不对称去芳构化反应

Figure 8 Asymmetric dearomatization reaction

我们利用不对称去芳构环化策略实现了具有全碳 手性季碳中心的三环化合物(雷公藤对醌类关键骨架)的 高效合成. 雷公藤对醌是从中药雷公藤中分离出来的一 类重要的天然产物，用于治疗发烧、发冷、浮肿等症状. 雷公藤对醌 $\mathrm{H}$ 作为代表性的雷公藤化合物之一，具有显 著的免疫抑制活性 ${ }^{[15]}$. 从结构上分析, 雷公藤对醌 $\mathrm{H}$ 属 于三环二萜苯醌结构. 其对苯醌类结构可以通过 $\mathbf{3 0}$ 的 芳基氧化得到(Scheme 10), 而含有手性季碳的并环结 构 31 可以通过不对称环化反应由芳基溴代物 32 得到. 一个季鏻盐 34 和醛 33 之间的 Wittig 反应可以实现环化 底物 32 的制备.

雷公藤对酮 $\mathrm{H}$ 的合成从醛 33 和季鏻盐 34 之间的 Wittig 反应开始(Scheme 11). 随后经双键还原和 TBS 保 护基脱除得到关环前体 32. 在 $\mathrm{Pd} / \mathrm{L} 3$ 催化条件下, 32 的 不对称去芳构环化顺利进行，我们以 $87 \%$ 的收率和 $85 \%$ 的 $e e$ 值得到含有季碳中心的环化产物 31 . 化合物 31 在 ${ }^{t} \mathrm{BuOK} / \mathrm{MeI}$ 的条件下实现了双键迁移和甲基化反应. 得 到烯酮 30 之后，再经过双键氢化和三溴化硼脱甲基得 到以反式/顺式异构体的混合物 35 (反式：顺式 $=1 ： 2$ ). 用弗雷米盐氧化后, 混合物 $\mathbf{3 5}$ 成功转化为雷公藤对醌 $\mathrm{H}$ 和 36. 两种产物可以很方便地通过制备高效液相色谱 分离. 


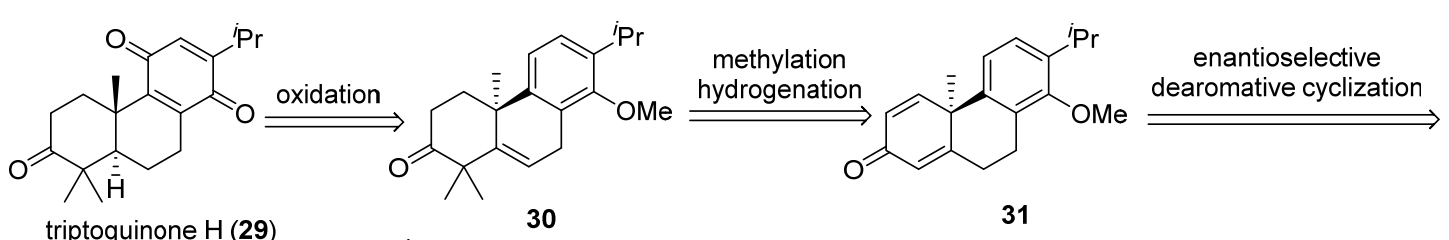

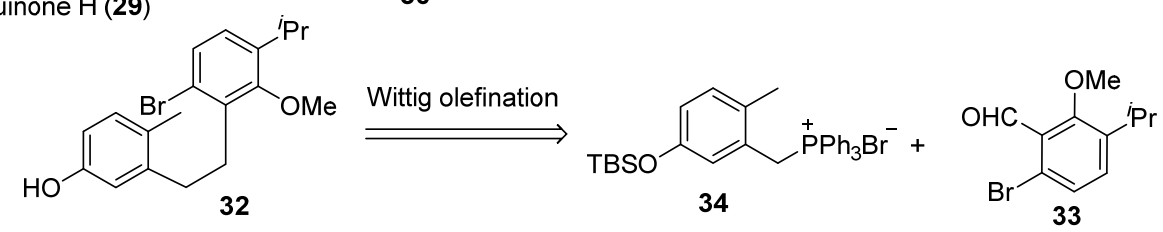

图式 10 雷公藤酮 $\mathrm{H}$ 的逆合成分析

Scheme 10 Retrosynthetic analysis of tripterone $\mathrm{H}$

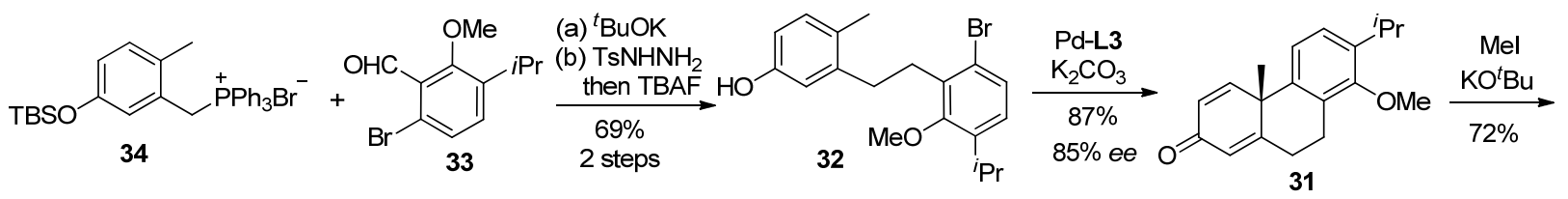<smiles>CCCc1ccc(C)c2c1CC=C1C(C)(C)C(=O)C=CC12C</smiles>

(a) $\mathrm{Pd} / \mathrm{C}, \mathrm{H}_{2}$
$\stackrel{\text { (b) } \mathrm{BBr}_{3}}{\mathrm{84 \%}}$
2 steps<smiles>CCCc1ccc2c(c1O)CC[C@@H]1C(C)(C)C(=O)CCC21C</smiles>
$\underset{90 \%}{\stackrel{\text { Frémy's salt }}{\longrightarrow}}$

$($ trans:cis $)=1: 2$

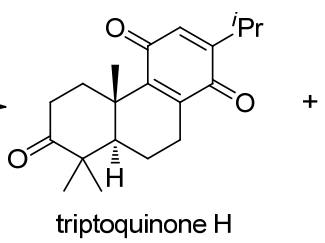<smiles>CCCC1=CC(=O)C2=C(CC[C@H]3[C@H](C)C(=O)CC[C@@]23C)C1=O</smiles>

36

$(29: \mathbf{3 6}=1: 2)$

图式 11 雷公藤对醌 $\mathrm{H}$ 的合成路线

Scheme 11 Synthetic route of tripterone $\mathrm{H}$

\section{4 不对称芳基烷基偶联合成布洛芬、氟比洛芬和萗} 普生

手性的 $\alpha$-芳基羰基化合物在有机合成中是一个非 常重要的中间体, 其通过多步转化可以合成诸多具有重 要生理活性的天然产物和药物分子. 对于这类结构的构 建，通常采用的策略是卤代芳烃与 $\alpha$-羊炭基金属试剂间的 反应，但该方法仅仅适用于活性较高的卤代芳烃. 其他 方法如使用当量的金属试剂与预制备的烯醇化合物反 应，则存在许多缺陷，如部分金属试剂对空气及水分敏 感、官能团兼容性差、使用的试剂高毒等. 因此, 发展 更高效的方法学实现低活性、种类广泛的芳基卤化物和 类卤化物或 $\alpha$-卤代羰基化合物参与的偶联反应迫在眉 睫. 对于前者, 过渡金属催化的 $\alpha$-芳基化反应已经取得 了长足的发展. 但由于该催化过程需要使用强碱, 反应 局限于季碳手性中心的构建, 这大大限制了该反应的适 用范围. 与之相比, 过渡金属催化 $\alpha$-卤代羰基化合物与 有机金属试剂的偶联反应条件更温和，且此类反应还可 以构建羰基 $\alpha$ 位的叔碳手性中心, 因而具有很大的发展 潜力和应用价值.

在过渡金属催化的 $\alpha$-卤代羰基化合物与有机金属 试剂, 如芳基硼酸的偶联反应的研究过程中, 我们发现 脱卤、 $\beta-\mathrm{H}$ 消除和异构化等副反应，这大大影响了这类
偶联反应的效率. 根据反应的机理， $\alpha$-卤代羰基化合物 经过零价钯物种氧化加成、转金属化后, 形成中间体 $C($ 图 9). 中间体 $C$ 可以直接还原消除得到我们所要的交 叉偶联产物, 或发生 $\beta-\mathrm{H}$ 消除以及经过第二次转金属产 生脱溴以及联芳基的副产物. 为了抑制副反应的发生, 我们提出发展大位阻的配体, 并在该配体中引入具有弱 配位能力的辅助基团, 如图 9 中的配体 $\mathbf{L}^{*}$. 我们设想此 类大位阻配体的空间环境迫使中间体 C 到 D 的过程不 易发生，从而有效地达到抑制二次转金属化的目的. 这 类 “ $\mathrm{P}, \mathrm{P}=\mathrm{O}$ ” 型配体中弱配位点 $\mathrm{P}=\mathrm{O}$ 的引人, 不仅便 于促进还原消除过程, 同时有效占有了钯中心周围的其 他配位位点，有效地抑制了 $\beta$ - $\mathrm{H}$ 消除、异构化和还原脱 卤等副反应. ${ }^{[16]}$

经过对配体结构的优化，我们发现手性配体 L4 可 以用于钯催化的 $\alpha$-溴代酰胺和芳基硼酸的不对称 Suzuki-Miyaura 交叉偶联反应中(Scheme 12). 利用 $\mathrm{Pd} /$ L4 催化的偶联反应可以方便地合成一系列手性的 $\alpha$-芳 基烷基酰胺类化合物.

手性的芳基丙酸类非甾体药物于 20 世纪 60 年代问 世. 这类药物具有高效的解热、镇痛和抗炎等作用, 其 中最有代表性的有布洛芬、䒺普生及氟比洛芬等. 布洛 芬于 1961 年由英国人 Stewart Adams 首次发现. 它主 


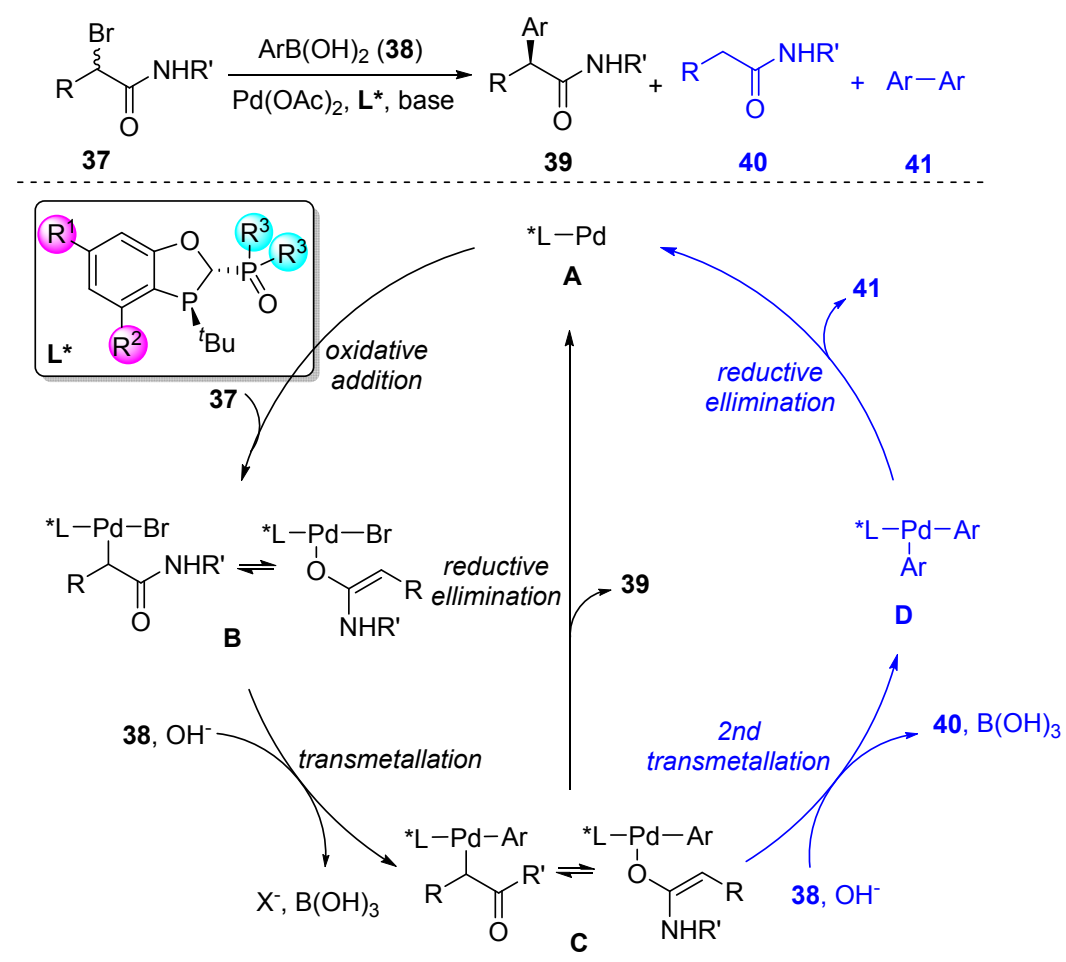

图 $9 \mathrm{Pd}$ 催化的烷基溴代物参与的 Suzuki-Miyaura 偶联反应

Figure 9 Pd-catalyzed Suzuki-Miyaura coupling using alkyl bromides

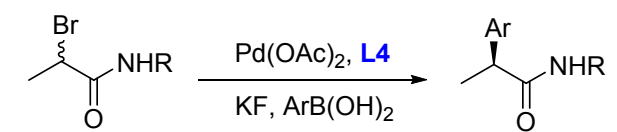

$\mathrm{R}=\mathrm{CH}\left(2-\mathrm{MeOC}_{6} \mathrm{H}_{4}\right)_{2}$

up to $94 \%$ ee $42 \% \sim 80 \%$ yield<smiles>CCCOc1cc(Nc2ccccc2)cc2c1P(Br)C(P(=O)([Ge])[Ge])O2</smiles>

L4

图式 $12 \mathrm{Pd}$ 催化的不对称芳基烷基偶联

Scheme 12 Pd-catalyzed asymmetric alkyl-aryl coupling

要用于发热、缓解轻度至中度的疼痛, 包括痛经、偏头 痛及类风湿关节炎等. 与布洛芬相比，氟比洛芬的毒性 更低并具有出色的耐受性, 其镇痛效果目前是丙酸类非 甾体药物中最强的. 菜普生是非甾体类抗炎药物, 除了 解热、镇痛等功效与布洛芬类似外, 䒺普生还可以提高 人体免疫力, 并对幽门螺杆菌有较好的抑制作用. 此外, 萗普生还具有调节血糖的作用 ${ }^{[17]}$. 通过钯催化的 $\alpha$-溴 代酰胺和芳基硼酸的不对称 Suzuki-Miyaura 交叉偶联反 应，可以很方便地合成布洛芬、氟比洛芬和萗普生 (Scheme 13). 以布洛芬的手性合成为例，芳基硼酸 43 和外消旋烷基溴代物 42 的交叉偶联得到高光学活性的 手性酰胺 44 (90\% ee). 将酰胺基团的胺基辅基脱除后可 以得到布洛芬. 相应地, 氟比洛芬和萗普生也可以通过 类似的合成方法高效制备.

\section{5 不对称芳基芳基偶联合成轴手性药物分子}

具有轴手性的联芳基结构广泛存在于药物和天然 产物分子中，例如临床上广泛使用的万古霉素类型的环 状多肽抗生素和具有包括抗肿瘤等活性的棉酚等. 目前 所报道的关于具有轴手性的联芳基化合物的合成方法 众多且各具特色. 在所有构建轴手性联芳基结构的策略 中，不对称偶联无疑是最为简洁且直接的方法. 通过不 对称偶联合成具有轴手性的联芳基结构，尤其是高度官 能团化的联芳基化合物的挑战在于提高偶联反应的反 应活性和对映选择性的高效调控. 在发展高立体选择性 的交叉偶联反应方面，我们通过设计新型配体和借助次 级相互作用的催化模式成功建立了用于合成邻位三取 代 ${ }^{[18]}$ 和四取代 ${ }^{[19]}$ 手性联芳基化合物的不对称 SuzukiMiyaura 偶联(图 10). 基于所发展的偶联反应，我们实现 了棉酚的不对称合成.

棉酚(gossypol)是一个多酚羟基双䒺醛类手性药物 分子. 这个化合物是由 Longmore 和 Marchlewski 两位科 学家于 19 世纪末从棉花籽油中首次分离得到 ${ }^{[19]}$. 棉酚 主要存在于棉花的根、茎、叶和种子内, 且在棉籽仁中 含量最高. 在中国，棉酚最早是用作男性避孕药来使用. 随着对其生物活性研究的深入，人们发现棉酚及其衍生 物具有多种生物活性，包括抗寄生虫、抗癌、抗病毒以 及抗感染等活性. 棉酚中的邻位四取代联芳基结构的存 


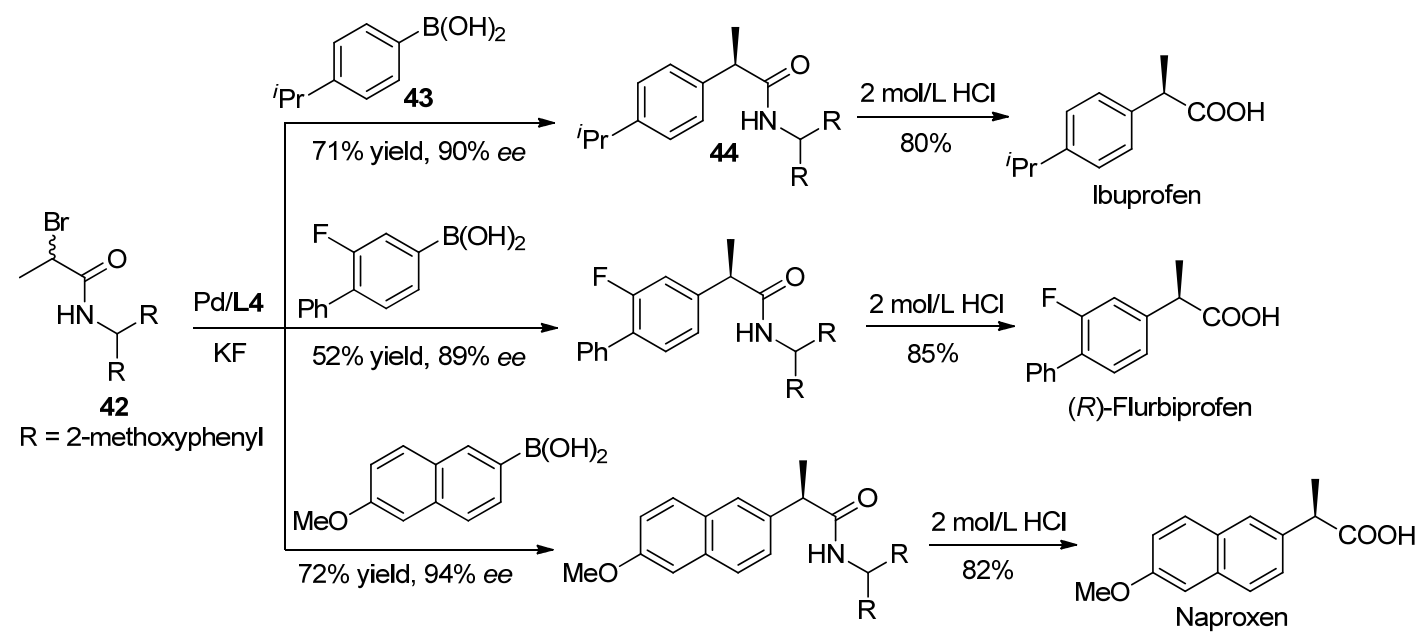

图式 13 布洛芬、氟比洛芬和萗普生的合成路线

Scheme 13 Asymmetric synthesis of ibuprofen, flurbiprofen, and naproxen

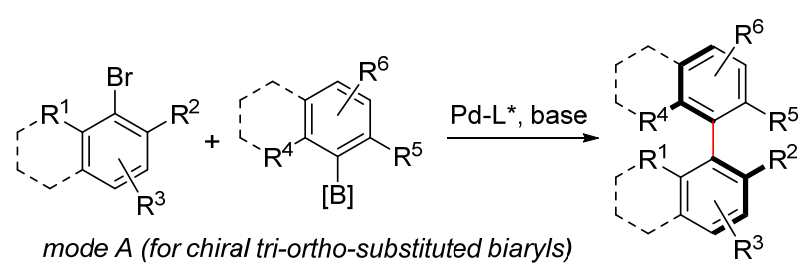$$
\text { *P--Pd-Ar' }
$$

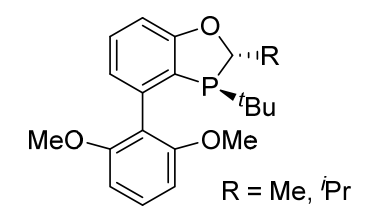

2nd interaction

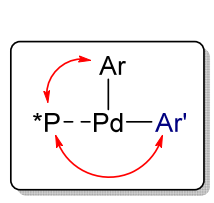

2nd interaction

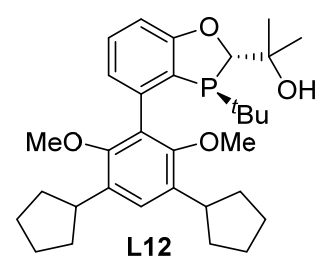

112
图 10 基于配体和催化模式设计的不对称 Suzuki-Miyaura 偶 联反应

Figure 10 Asymmetric Suzuki-Miyaura coupling based on design of ligand and catalysis mode

在使其沿联菜基碳碳键的旋转具有一定的能垒，因而该 分子具有轴手性. 从棉花中提取分离得到的棉酚往往是 两个对映体 $(+)-(S)$-棉酚和 $(-)-(R)$-棉酚的混合物. 在 生物活性方面, (十)-棉酚、(一)-棉酚以及它们的混合物 也会有一定的差别, 例如, 棉酚的消旋体具有抗生育活 性，而(十)-棉酚则发现不具备这一活性; (一)-棉酚或者 含有较高比例的 (一)-棉酚棉籽油具有抑制乳腺脂肪细 胞增殖的活性, 因而具有抗乳腺癌和预防肥胖的潜 力 $^{[31]}$; 另有研究表明, (一)-棉酚可以在 mRNA 和蛋白质 水平上干预促血管生成因子从癌细胞的释放过程, 因而 可以抑制肿瘤血管生成过程. 正是由于棉酚两个对映体
在生物活性方面的差异, 发展能够得到高光学纯度的单 一棉酚对映体的方法至关重要. 目前已报道且普遍采用 的获得高光学纯度的单一棉酚对映体的方法是化学拆 分法. 此方法通过棉酚的消旋体和手性拆分试剂(通常 是手性氨基酸)作用生成相应的席夫碱, 然后通过高效 液相色谱法或重结晶方法获得单一的立体异构体，再经 过拆分试剂的脱除得到高光学纯度的单一棉酚对映体. 此方法的局限性在于当量手性拆分试剂的使用, 这无疑 增加了整个过程的成本; 同时，棉酚消旋体的获得也是 该方法规模化的一个制约因素. 相比较而言, 利用廉价 易得的原料，通过化学合成的方法来得到高光学纯度的 单一棉酚对映体更有前景. 至今为止, 通过不对称合成 的方法得到高光学纯度的单一棉酚对映体目前仅有一 例报道，即 Meyers 课题组利用手性辅基底物参与的乌 尔曼偶联反应为关键步骤实现的棉酚的不对称全合成. 手性辅基的使用增加了生产的成本; 同时，手性辅基的 引入和脱除也使得合成路线不够简洁实用. 因而发展一 种简洁高效地获得高光学纯度的单一棉酚对映体的化 学合成方法有着重要的意义.

根据对棉酚的逆合成分析, 我们认为其高度官能团 化的联芳基结构可以由前体 45 通过后期的异丙基化, 脱保护基和甲酰化反应实现(Scheme 14). 轴手性联芳 基结构则可以利用分子间的不对称 Suzuki-Miyaura 偶联 构建. 取代的萗基底物可以有萗醌前体的选择性还原得 到. 醌类化合物 49 和二烯 48 参与的环加成反应可以合 成蒜醌 47.

我们发展了如 Scheme 15 所示的棉酚的不对称合成 路线. 首先已知化合物共轭二烯 48 与苯醌化合物 49 发 生环加成反应, 制得菜醌化合物. 将菜醌化合物进行选 择性地还原并原位将酚羟基保护得到取代的䒺化合物 47. 在溴化氧化条件下将化合物 47 转化为菱甲醛 $\mathbf{5 1}$, 

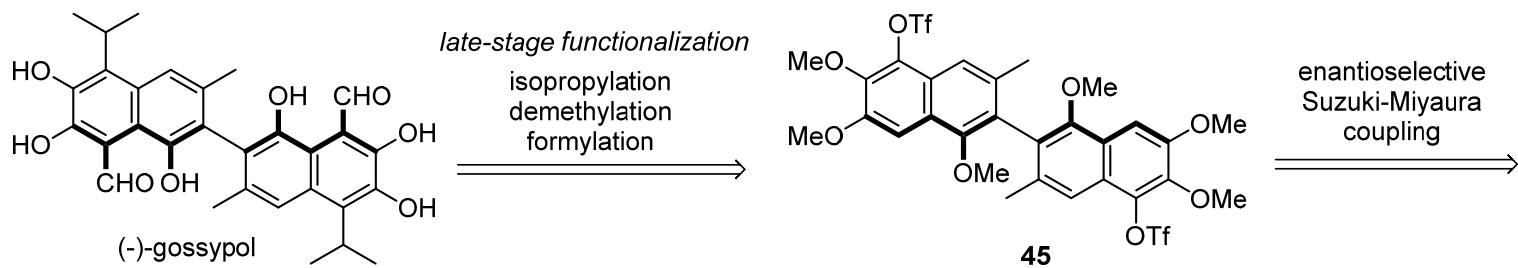<smiles>[Z17]Oc1cc2c(OC)c(Br)c(C=O)cc2c(OC)c1OC</smiles>

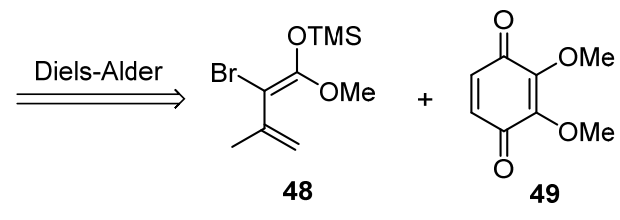

图式 14 棉酚的逆合成分析

Scheme 14 Retrosynthetic analysis of gossypol<smiles>CCOC(=O)OC1=C(OC)C(=O)C=CC1=O</smiles><smiles></smiles><smiles>COc1cc2c(OC)c(-c3c(OC)cc4c(OC)c(OC)c(OC)cc4c3OC)c(C)cc2c(OC)c1OC</smiles>

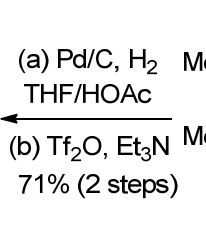<smiles>COc1ccc(OC)cc1</smiles><smiles>COc1cc2c(OC)c(OC)c(OC)c(-c3c(C)cc4c(OC)c(OC)ccc4c3OC)c2c(OC)c1OC</smiles>

$\mathrm{Pd}_{2}(\mathrm{dba})_{3}(2.5 \mathrm{~mol} \%)$ $(R, R)$-BaryPhos (L12)

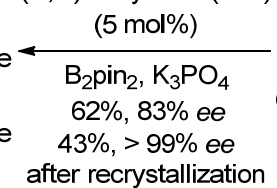<smiles></smiles>

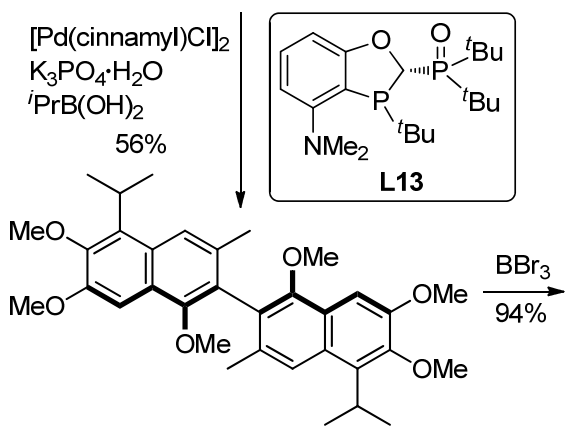

55 (-)-gossypol hexamethyl ether<smiles>COc1c(O)c(O)c(C(C)C)c2cc(O)c(-c3c(C)cc4c(C(C)C)c(O)c(O)cc4c3O)c(O)c12</smiles><smiles>Cc1cc2c(C(C)C)c(O)c(O)c(C=O)c2c(O)c1-c1c(C)cc2c(C(C)C)c(O)c(O)c(CO)c2c1O</smiles>

图式 15 棉酚的对映选择性合成

Scheme 15 Enantioselective synthesis of gossypol

同时脱除菜酚差基上的乙酰基保护基得到 51.51 参与的 烷基化反应得到偶联前体 52. 在过渡金属钯催化剂和 手性配体 BaryPhos (L12)的存在下, 化合物 52 进行 Miyaura 硼化反应, 并进一步发生 Suzuki-Miyaura 偶联 反应，以 $62 \%$ 的产率和 $83 \% \mathrm{ee}$ 制得具有轴手性的联芳 基化合物 53. 通过重结晶可以进一步提高该联芳基化 合物的光学纯度 $(>99 \% e e)$. 得到 $\mathbf{5 3}$ 后, 催化氢解和三 氟甲磺酰化制得 54, 接着芳基异丙基偶联在 $\mathrm{Pd} / \mathrm{L} 13$ 催 化剂存在下顺利进行得到 55. 最后脱甲基和甲酰化反 应制得(一)-棉酚.

手性联芳基化合物 57 是基于喹啉结构发展而来的
具有别构效应的整合酶抑制剂，是旨在解决获得性免疫 缺陷综合征(艾滋病)治疗过程中药物耐药性问题的一个 潜在药物(Scheme 16). 除了轴手性联芳基结构外, 这个 化合物分子内还含有一个手性仲醇的叔丁醚单元. 为了 发展一条高效简洁的合成路线，我们认为其联芳基结构 可以通过 58 和 59 参与的不对称 Suzuki-Miyaura 交叉偶 联构建 ${ }^{[21,22]}$. 通过使用合适的配体，可以实现对该偶联 反应的立体选择性的调控. 手性二级醇可以经过相应酮 前体 60 的不对称还原合成. 喹啉衍生物 61 经酰基化反 应可以合成得到 60.

如 Scheme 17 所示, 由已知化合物 61 出发, 经过碘 


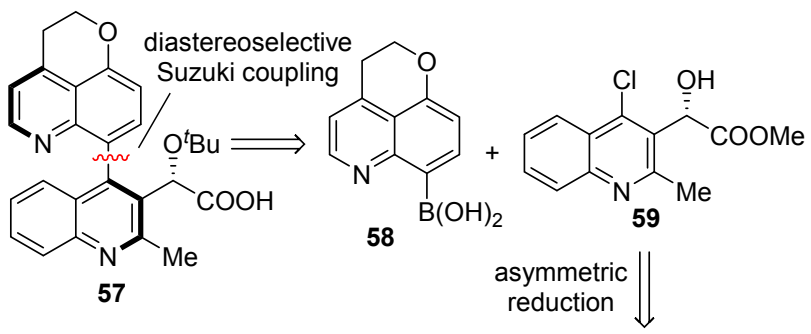<smiles>COC(=O)c1c(C)nc2ccccc2c1Cl</smiles>

图式 16 药物分子 $\mathbf{5 7}$ 的逆合成分析 Scheme 16 Retrosynthetic analysis of 57

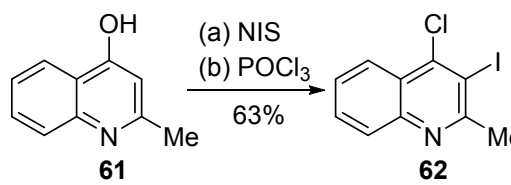<smiles>COC(=O)C(=O)OC</smiles>
$79 \%$<smiles>CC(=O)OC(=O)c1c(C)nc2ccccc2c1Cl</smiles>
60<smiles>NC(c1ccccc1)[C@@H](NS(=O)(=O)c1ccc([N+](=O)[O-])cc1)c1ccccc1</smiles>
$87 \%, 98 \%$ ee<smiles>COc1ccc(Br)c(N)c1</smiles>
63 64

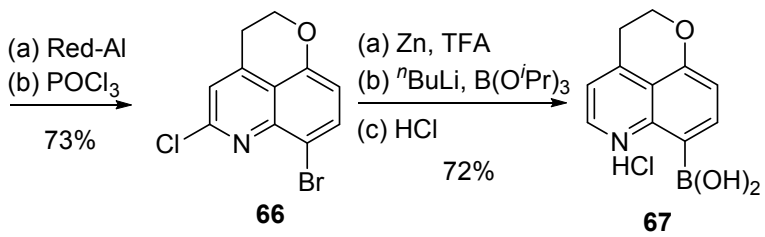

图式 17 关键中间体的合成

Scheme 17 Synthesis of key intermediates of 49

代和氯化反应可以合成 62.62 经过金属卤交换后与酰氯 反应, 得到酰化产物 60. 随后在 $\mathrm{Rh} / \mathrm{L14}$ 的催化下, 60 经 过不对称氢化反应合成得到手性醇 59. 芳基嗍酸 58 的 合成是由 63 和 64 作为起始原料, 在不饱和酸䣶 64 的存 在下, 苯胺 63 首先发生酰基化反应, 随后在浓硫酸条件 下发生关环反应和酯化反应得到酯 65.65 在 Red-Al 条 件下被还原为相应的醇. 其经过 $\mathrm{POCl}_{3}$ 介导的环化和锌 粉还原脱氯转化为芳基溴 66. 最后在正丁基锂/硼酸三
异丙酯条件下, 66 转化为相应的嗍酸盐酸盐 67.

作为合成的关键步骤, $\mathrm{Pd} / \mathbf{L 1}$ 催化的 $\mathbf{5 8}$ 和 $\mathbf{5 9}$ 的 Suzuki-Miyaura 交叉偶联反应可以以 $73 \%$ 的收率和 $5: 1$ 的 $d r$ 值得到目标产物 68 (Scheme 18). 进一步的配体结 构优化提高了偶联反应的立体选择性, 即当使用配体 $\mathbf{L} 15$ 时, 反应的 $d r$ 值可以提升至 $15: 1$. 值得指出的是, 通过重结晶可以将手性醇 $\mathbf{6 8}$ 的光学纯度提升至 $99 \%$ ee. 最后经过改进的 Jackson 叔丁基化和酯的水解合成得到 目标产物 57.
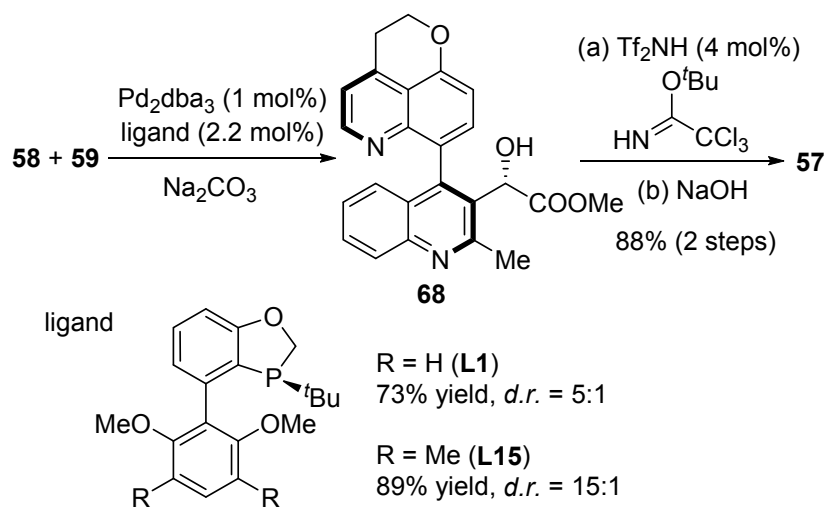

图式 18 化合物 57 的不对称合成

Scheme 18 Asymmetric synthesis of 57

\section{3 总结与展望}

综上所述, 发展新型高效的不对称合成方法对手性 药物的简洁合成具有重要意义. 近年来, 手性配体促进 的过渡金属催化的不对称转化在手性药物合成中的应 用越来越广泛. 本文介绍了基于苯并手性氧杂膦烷结构 单元的单、双膦配体的设计理念, 并详细总结了这些具 有独特骨架的配体在过渡金属催化的不对称偶联、环 化、氢化和加成等反应中的应用. 利用所发展的不对称 反应方法学，我们实现了包括托法替尼、cipargamin、艾 司西酞普兰、雷公藤对醌 $\mathrm{H}$ 、布洛芬、氟比洛芬、菜普 生和棉酚在内的一系列重要手性药物分子的高效不对 称合成. 可以预见, 未来新型手性配体/催化剂和不对称 方法学的不断发展必将进一步促进手性药物的绿色合 成.

\section{作者简介}

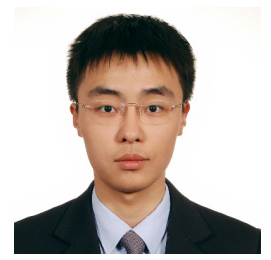

许容华, 博士研究生. 2017 年本科毕业于上海师范 大学生命与环境科学学院(现化学与材料科学学院)化学 
化工系化学(师范)专业, 现为上海科技大学与中国科学 院上海有机化学研究所联合培养在读博士研究生(导师: 汤文军研究员), 主要从事手性膦配体的设计与合成以 及其催化的不对称反应研究.

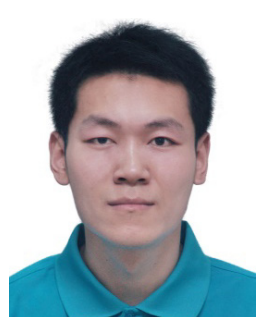

杨贺, 博士, 2008 年本科毕业于山东大学化学与化 工学院, 2011 年于山东大学化学与化工学院获硕士学位 (导师: 王建武教授), 2015 年于英国伯明翰大学获博士 学位(导师: Nigel Simpkins 教授). 2016 2019 年在中国 科学院上海有机化学研究所从事博士后研究(导师: 汤 文军研究员). 2019 年 9 月入职南方科技大学深圳格拉布 斯研究院高级研究学者职位. 主要研究方向为: 新型手 性配体和不对称催化反应的发展.

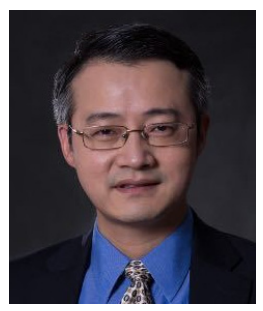

汤文军, 博士, 1995 年毕业于华东理工大学精细化 工系, 1998 年于中国科学院上海有机化学研究所获硕士 学位(导师: 马大为院士); 2003 年于美国宾夕法尼亚州 立大学获博士学位(导师: 张绪穆教授). 2003 2005 年 在美国 Scripps 研究所从事博士后研究(导师: K. C. Nicolaou 教授), 2005 2009年在美国 Boehringer Ingelheim 药物工艺部门任 Senior Scientist, 2009～2011 年任 Principal Scientist, 2011 年 7 月起任中国科学院上海有机 化学研究所研究员、课题组长. 同时兼任上海科技大学 物质学院教授、博士生导师及华东理工大学药学院博士 生导师, 2012 年获国家 “青年千人计划” 基金资助, 2015 年获中国均相催化青年奖, 2017 年获国家杰出青年基金 资助. 2018 年入选科技部中青年科技创新领军人才, 2019 年入选科技部 “万人计划”. 主要研究方向为: 不 对称催化、天然产物全合成、药物工艺.

\section{References}

[1] Lin, G. Q.; Wang, M. X. Chiral Synthesis and Chiral Drugs, Chemical Industry Press, Beijing, 2008, pp. 1 4 (in Chinese). (林国强, 王梅祥, 手性合成和手性药物, 化学工业出版社, 北 京, 2008, pp. 1 4.)

[2] Lin, G. Q.; Sun, X. W.; Chen, Y. Q.; Li, Y. M.; Chen, X. Z. Chiral Synthesis-Asymmetric Reactions and Their Applications, Science Press, Beijing, 2013, pp. 36 38 (in Chinese).

(林国强, 孙兴文, 陈耀全, 李月明, 陈新滋, 手性合成一一不对 称反应及其应用, 科学出版社, 北京, 2013, pp. 36 38.)

[3] Tang, W; Zhang, X. Chem. Rev. 2003, 103, 3029.

[4] Tang, W. In Proceedings of the 11th National Conference on Natural Organic Chemistry of the Chinese Chemical Society, Volume 1, Shanghai, 2019, p. 71 (in Chinese).

(汤文军, 中国化学会第十一届全国天然有机化学学术会议论文 集, 第一册, 上海, 2019, p. 71.)

[5] (a) Xu, G.; Senanayake, C. H; Tang, W. Acc. Chem. Res. 2019, 52, 1601.

(b) Wu, T.; Xu, G.; Tang, W. Aldrichim. Acta 2020, 53, 27.

(c) Tang, W.; Li, K. Strem Chem. 2019, XXXI, 1.

[6] Liu, G.; Liu, X.; Cai, Z.; Jiao, G.; Xu, G.; Tang, W. Angew. Chem., Int. Ed. 2013, 52, 42359.

[7] Li, C.; Wan, F; Chen, Y.; Peng, H.; Tang, W.; Yu, S.; McWilliams, H. C.; Mustakis, J.; Samp, L.; Maguire, R. J. Angew. Chem., Int. Ed. 2019, 58, 13573.

[8] Daniella M. S.; Yuka K.; Alejandro V.; Michael W.; Massimo G.; John J. O. Nat. Rev. Drug Discovery 2017, 16, 843.

[9] Patil, Y. S.; Bonde, N. L.; Kekan, A. S.; Sathe, D. G.; Das, A. Org. Process Res. Dev. 2014, 18, 1714.

[10] Zhu, J.; Huang, L.; Dong, W.; Li, N.; Yu, X.; Deng, W.; Tang, W. Angew. Chem., Int. Ed. 2019, 58, 16119.

[11] Rottmann, M.; McNamara, C.; Yeung, B. K. S. Science 2010, 329, 1175.

[12] Huang, L.; Zhu, J.; Jiao, G.; Wang, Z.; Yu, X.; Deng, W.; Tang W. Angew. Chem., Int. Ed. 2016, 55, 4527.

[13] Dhillon, S.; Scott, L. J.; Plosker, G. L. CNS Drugs 2006, $20,763$.

[14] Yang, H.; Tang, W. Chem. Rec. 2020, 20, 23.

[15] Cao, Z.; Du, K.; Liu, J. H.; Tang, W. Tetrahedron 2016, 72, 1782.

[16] Li, B.; Li, T.; Aliyu, M. A.; Li, Z.; Tang, W. Angew. Chem., Int. Ed. 2019, 58,11355 .

[17] Dodou, K.; Anderson, R. J.; Lough, W. J.; Small, D. A. P.; Shelley, M. D.; Groundwater, P. W. Bioorg. Med. Chem. 2005, 13, 4228.

[18] (a) Xu, G.; Fu, W.; Liu, G.; Senanayake, C. H.; Tang, W. J. Am. Chem. Soc. 2014, 136, 570 .

(b) Xu, G.; Zhao, Q.; Tang, W. Chin. J. Org. Chem. 2014, 34, 1919 (in Chinese).

(徐广庆, 赵庆, 汤文军, 有机化学, 2014, 34, 1919.)

[19] Yang, H.; Sun, J.; Gu, W.; Tang, W. J. Am. Chem. Soc. 2020, 142, 8036.

[20] Lu, Y.; Dong, C.; Huang, J.; Zhou, H.; Wang, W. Future Med. Chem. 2017, 9, 1243.

[21] Fandrick, K. R.; Li, W.; Zhang, Y.; Tang, W.; Gao, J.; Rodriguez, S.; Patel, N. D.; Reeves, D. C.; Wu, J.-P.; Sanyal, S.; Gonnella, N.; Qu, B.; Haddad, N.; Lorenz, J. C.; Sidhu, K.; Wang, J.; Ma, S.; Grinberg, N.; Lee, H.; Tsantrizos, Y.; Poupart, M.-A.; Busacca, C. A.; Yee, N. K.; Lu, B. Z.; Senanayake, C. H. Angew. Chem., Int. Ed. 2015, 54, 7144.

[22] Haddad, N.; Mangunuru, H. P. R.; Fandrick, K. R.; Qu, B.; Sieber, J. D.; Rodriguez, S.; Desrosiers, J. N.; Patel, N. D.; Lee, H.; Kurouski, D.; Grinberg, N.; Yee, N. K.; Song, J. J.; Senanayake, C. H. Adv. Synth. Catal. 2016, 358, 3522. 